\title{
Effect of Physical and Flexibility Exercise on Plasma Levels of Some Liver Enzymes and Biomolecules of Young Nigerian Adults
}

\author{
PO Uadia 1 , KO Orumwensodia ${ }^{1}$, GE Arainru ${ }^{2}$, EO Agwubike ${ }^{2}$ and CBN Akpata ${ }^{1}$ \\ ${ }^{1}$ Department of Biochemistry, ${ }^{2}$ Department of Human Kinetics and Sports Science, University of Benin, Benin City, Nigeria
}

*For correspondence: Email: psouadia@uniben.edu; Tel: +234-8055783197

Received: 6 May 2015

Revised accepted: 4 January 2016

\begin{abstract}
Purpose: To evaluate the effect of physical and flexibility exercise on plasma levels of some liver enzymes and biomolecules of young Nigerian adults.

Methods: Participants were subjected to a 2-h daily continuous physical and flexibility exercise for 6 weeks. Pre- and post-exercise blood samples were obtained and the effect of exercise on plasma levels of alanine aminotransferase (ALT), aspartate aminotransferase (AST), alkaline phosphatase (ALP), lactate dehydrogenase (LDH), gamma glutamyl transferase (GGT), total protein, albumin and bilirubin, were determined.

Results: AST and LDH values increased significantly $(p<0.05)$ in males, while only AST values increased significantly $(p<0.05)$ in females post-exercise. ALT, ALP, GGT and bilirubin values for both sexes were not significantly $(p>0.05)$ altered post-exercise. Post-exercise values of total protein and albumin decreased significantly $(p<0.05)$ in males, but not in females.

Conclusion: The 6-week physical and flexibility exercise did not substantially affect the plasma levels of some liver enzymes and the composition of other biomolecules.
\end{abstract}

Keywords: Physical and flexibility exercise, Liver enzymes, Biochemical profile

Tropical Journal of Pharmaceutical Research is indexed by Science Citation Index (SciSearch), Scopus, International Pharmaceutical Abstract, Chemical Abstracts, Embase, Index Copernicus, EBSCO, African Index Medicus, JournalSeek, Journal Citation Reports/Science Edition, Directory of Open Access Journals (DOAJ), African Journal Online, Bioline International, Open-J-Gate and Pharmacy Abstracts

\section{INTRODUCTION}

Exercise is defined as bodily movement produced by the contraction of skeletal muscle that substantially increases energy expenditure [1]. Thompson et al [2], also defined exercise or physical activity as any bodily movement produced by skeletal muscles that results in energy usage beyond resting energy expenditure. From these definitions, it is clear that physical activity exerts the body muscles and involves the expenditure of energy in form of ATP. Depending on the overall effect physical exercise has on the body, it is grouped into three types; aerobic (endurance), anaerobic (resistant) and flexibility exercise [1,3-5]. The immense benefits from exercising include enhanced mood, self-esteem and improved physical appearance. Also, exercise helps in the reduction of several diseases such as cancers, cardiovascular diseases, non-insulin dependent diabetes (type II diabetes), osteoporosis and ultimately reduction in premature mortality [6]. Again, exercise aids cardiovascular and respiratory functions, slows the loss of muscular strength thereby enhancing muscle tone, increases bone mass, slows the process of aging, aids digestion and bowel function, promotes sound sleep and prevents depression [6]. These benefits would depend on the type, intensity, duration and frequency of 
exercise programme, and the characteristics of the individual at baseline [7]. However, the benefits of exercising are transient and reversible [8].

Hence, in a bid to perform maximally and ensure survival, the body tends to adjust to strain imposed on it by stress such as physical exercise, thus, affecting the body's anatomical, physiological as well as its metabolic state [9]. Nonetheless, some forms of exercise and particularly strenuous exercise are also accompanied by some deleterious effects. In this context, muscle damage post exercise leads to substantial increase in myocellular protein levels in the blood $[10,11]$. It has been shown that prolonged physical exercise results in transient elevations of biochemical markers of muscular damage such as aspartate aminotransferase (AST), alanine aminotransferase (ALT), creatine kinase (CK), and lactate dehydrogenase (LDH) [12]. Therefore and in agreement with Bray [13], the benefits from exercising is maximized when exercises are directed at meeting the individual's needs and capacity.

The objective of this study was to evaluate the effect of physical and flexibility exercise on plasma levels of some liver enzymes and other biomolecules in apparently healthy young Nigerian adults.

\section{EXPERIMENTAL}

\section{Subjects}

Sixteen (16) young Nigerian adult students who were apparently healthy and not on any exercise regimen were used for this study. They included eleven (11) males, aged $25.40 \pm 1.09$ years and five (5) females, aged $22.83 \pm 0.83$ years. Participants were instructed to maintain their daily dietary habits for the duration of the research period and avoid taking medications such as pain relievers.

\section{Exercise program}

The study was carried out four (4) times a week (Monday, Wednesday, Friday and Saturday), between the hours of 5:30 and 7:30 am, for a period of six (6) weeks. Each participant covered a total distance of $56,500 \mathrm{~m}$. The exercise regimen which was mainly aerobic began with jogging of varied but specified distances and immediately followed by various flexibility exercises (calisthenics, which included; sit ups, neck rotations (left-right, back-forward), arm and waist rotations (left-right, back-forward), press- up, Limbs stretches, imaginary cycling-with the back lying on the floor, etc.).

\section{Blood sampling and analysis}

Pre-exercise (24 $\mathrm{h}$ before exercise) and post exercise (24 h after exercise) intravenous blood samples were obtained from the participants (at a sitting position) in the morning after a 12-h fast. About $10 \mathrm{ml}$ blood sample was collected into heparinized bottles before and after the exercise. Samples were immediately placed in an ice bath and centrifuged at $2500 \mathrm{rpm}$ for $10 \mathrm{~min}$. Aliquots of the resulting plasma were stored at $-80^{\circ} \mathrm{C}$ until analyzed. All enzyme assays were done using the Selectra ProS auto analyzer (Elitech Group, France) and reagent kits were procured from Elitech clinical systems (Elitech Group, France). Albumin, total protein and bilirubin were determined spectrophotometrically as described by Randox reagent kit, (Randox, UK).

The measurement of serum albumin was based on its quantitative binding to the indicator 3,3 ', 5 , 5'- tetrabromo - $n$ cresol sulphonephthalein (bromocresol green, BCG). Albumin -BCG complex absorbs maximally at $578 \mathrm{~nm}$, the absorbance being directly proportional to the concentration of albumin in the sample. Total protein was estimated by the Biuret method, wherein, cupric ions in an alkaline medium interact with protein peptide bonds resulting in the formation of a coloured complex which is read spectrophotometrically at $546 \mathrm{~nm}$. Direct (conjugated) bilirubin reacts with diazotized sulphanilic acid in alkaline medium to form a blue colored complex. Total bilirubin is determined in the presence of caffeine, which releases albumin bound bilirubin, by the reaction with diazotized sulphanilic acid. Both absorbance are read at $578 \mathrm{~nm}$. While, indirect bilirubin is the difference between total and direct bilirubin.

\section{Statistical analysis}

All statistical analyses were carried out using Microsoft Excel (Windows 8.0). Descriptive statistics are presented as mean and standard error of mean. Comparison of continuous variables was done using Student paired t-test. $P$ $<0.05$ was considered statistically significant.

\section{RESULTS}

\section{Enzyme profile}

The results of the effect of six (6) weeks physical and flexibility exercise on plasma levels of liver enzymes' activity of male and female participants are presented in Tables 1 and 2 below. 
Table 1 shows that only AST and LDH values showed significant $(p<0.05)$ increase postexercise. However, there was a non-significant $(p$ $>0.05$ ) decrease in the values of ALT, ALP and GGT post-exercise.

Table 2 indicate that ALT, AST and GGT increased post-exercise, but the increase was only significant for AST post-exercise; ALP and $\mathrm{LDH}$ values decreased though not significantly $(p$ $>0.05$ ) post-exercise. However, all values were within the reference range.

\section{Biochemical profile}

The effect of six (6) weeks physical and flexibility exercise on plasma levels of some biochemical parameters of both sexes, was tested and shown in Tables 3 and 4 below.

There was an insignificant $(p>0.05)$ decrease in the bilirubin values post-exercise as seen from Table 3. However, a significant $(p<0.05)$ decrease in the post-exercise results for total protein and albumin was also observed.

Table 1: Effect of six (6) weeks physical and flexibility exercise on plasma levels of some liver enzymes' activity in male participants

\begin{tabular}{lccccc}
\hline Variable & ALT (U/L) & AST (U/L) & ALP (U/L) & LDH (U/L) & GGT (U/L) \\
\hline Pre-exercise & $22.4 \pm 4.02$ & $20.90 \pm 1.58$ & $174.10 \pm 14.20$ & $203.90 \pm 11.59$ & $32.00 \pm 5.73$ \\
\hline Post-exercise & $21.9 \pm 2.34$ & $25.70 \pm 1.01^{*}$ & $153.80 \pm 14.08$ & $240.40 \pm 9.26^{*}$ & $24.40 \pm 2.75$ \\
\hline Reference value & $\leq 45$ & $<40$ & $<270$ & $<248$ & $<55$ \\
\hline
\end{tabular}

Data represent mean \pm SEM ( $n=10, p=0.05$, paired $t$-test); $n=$ number of male participants; $A L T=$ alanine aminotransferase, $A S T=$ aspartate aminotransferase, $A L P=$ alkaline phosphatase, $L D H=$ lactate dehydrogenase, and GGT = $\gamma$-glutamyl transferase; * represent significant increase $(p<0.05)$ compared with pre-exercise value

Table 2: Effect of six-week physical and flexibility exercise on the plasma levels of some liver enzyme activities in female participants

\begin{tabular}{lllccc}
\hline Variable & ALT (U/L) & AST (U/L) & ALP (U/L) & LDH (U/L) & GGT (U/L) \\
\hline Pre-exercise & $12.33 \pm 1.31$ & $18.83 \pm 1.14$ & $194.17 \pm 22.98$ & $219.00 \pm 13.15$ & $19.50 \pm 3.35$ \\
\hline Post-exercise & $25.50 \pm 6.08$ & $26.67 \pm 2.79^{*}$ & $187.50 \pm 19.63$ & $216.67 \pm 17.08$ & $28.50 \pm 8.23$ \\
Reference & $\leq 34$ & $<40$ & $\leq 270$ & $<248$ & $32-46$ \\
\hline
\end{tabular}

Data represent mean \pm SEM ( $n=6, p=0.05$, paired $t$-test); $n=$ number of female participants; $A L T=$ alanine aminotransferase, $A S T=$ aspartate aminotransferase, $A L P=$ alkaline phosphatase, $L D H=$ lactate dehydrogenase, and GGT = $\gamma$-glutamyl transferase; * represent significant increase $(p<0.05)$ compared with pre-exercise value

Table 3: Effect of six-week physical and flexibility exercise on plasma levels of some biochemical parameters of male participants

\begin{tabular}{lccccc}
\hline Variable & $\begin{array}{c}\text { Total bilirubin } \\
(\mathbf{m} \mathbf{g} / \mathbf{d} \mathbf{l})\end{array}$ & $\begin{array}{c}\text { Direct bilirubin } \\
(\mathbf{m g} / \mathbf{d l})\end{array}$ & $\begin{array}{c}\text { Indirect } \\
\text { bilirubin } \\
(\mathbf{m g} / \mathbf{d} \mathbf{l})\end{array}$ & $\begin{array}{c}\text { Total protein } \\
(\mathbf{g} / \mathbf{d l})\end{array}$ & $\begin{array}{c}\text { Albumin } \\
(\mathbf{g} / \mathbf{d l})\end{array}$ \\
\hline Pre-exercise & $0.82 \pm 0.15$ & $0.51 \pm 0.09$ & $0.33 \pm 0.10$ & $8.29 \pm 0.16$ & $4.93 \pm 0.11$ \\
\hline Post-exercise & $0.82 \pm 0.12$ & $0.49 \pm 0.07$ & $0.33 \pm 0.06$ & $7.56 \pm 0.15^{\star}$ & $4.60 \pm 0.06^{*}$ \\
\hline Reference value & $0.93-1.35$ & $0.49-0.81$ & $0.44-0.54$ & $6.06-7.72$ & $3.5-4.5$ \\
\hline Data represent mean $\pm S E M\left(n=10, p=0.05\right.$, paired $t$-test); $n=$ number of male participants; ${ }^{*}$ represent
\end{tabular}
significant decrease $(p<0.05)$ compared with pre-exercise value

Table 4: Effect of six-week physical and flexibility exercise on plasma levels of some biochemical parameters of female participants

\begin{tabular}{lccccc}
\hline Variable & $\begin{array}{c}\text { Total bilirubin } \\
(\mathbf{m g} / \mathbf{d l})\end{array}$ & $\begin{array}{c}\text { Direct bilirubin } \\
\mathbf{( m g / d l )}\end{array}$ & $\begin{array}{c}\text { Indirect } \\
\text { bilirubin } \\
(\mathbf{m g} / \mathbf{d l})\end{array}$ & $\begin{array}{c}\text { Total protein } \\
\mathbf{( g / d l )}\end{array}$ & Albumin (g/dl) \\
\hline Pre-exercise & $0.58 \pm 0.07$ & $0.38 \pm 0.06$ & $0.20 \pm 0.03$ & $8.43 \pm 0.24$ & $4.87 \pm 0.19$ \\
\hline Post-exercise & $0.42 \pm 0.05$ & $0.30 \pm 0.03$ & $0.13 \pm 0.02$ & $7.97 \pm 0.11$ & $4.70 \pm 0.08$ \\
\hline Reference value & $0.93-1.35$ & $0.49-0.81$ & $0.44-0.54$ & $6.06-7.72$ & $3.5-4.5$ \\
\hline
\end{tabular}

Data represent mean \pm SEM $\left(n=6, p=0.05\right.$, paired t-test); $n=$ number of female participants; ${ }^{*}$ represent significant decrease $(p<0.05)$ compared with pre-exercise value 
From the results presented in Table 4, there was no significant $(p>0.05)$ difference in the results of all female participants. Although, there was a general decrease in post-exercise values.

\section{DISCUSSION}

The efficacy of physical exercise in health management and disease control has been suggested for the sustenance of healthy living. This study provides some scientific data to support this concept. The results obtained from this study indicate that physical exercise could be of immense benefit to an individual's health. Alanine aminotransferase (ALT) which is predominantly found in the liver, but with smaller amounts in the kidneys, heart, muscles, pancreas, and used as an indicator for liver injury, showed a decrease in the post-exercise values as against the pre-exercise values, although this decrease was not statistically significant $(p>0.05)$. This suggests that the pattern, intensity and duration of the exercise regimen did not cause damage to the liver which is the major source of ALT. This is in agreement with Davries et al [14]. A similar result was reported by Sreenivasa et al [15], after subjecting patients with non-alcoholic steatohepatitis to exercise, while Nuri et al [16], reported a significant decrease in serum ALT values in postmenopausal women with breast cancer who were subjected to a fifteen week exercise regimen.

AST is not as specific an indicator of liver damage as ALT because of its multi-organ distribution. Therefore, stressors such as physical exercise could impact negatively on the heart and skeletal muscles, thus leading to the release of AST into the bloodstream, which eventually leads to increase in serum AST levels [17]. This was the trend noticed when the postexercise results of AST for both male and female participants were considered. The results presented in Tables 1 and 2, showed a significant $(p<0.05)$ increase in AST values after the six weeks physical and flexibility exercise. The sources of the AST are likely from injured muscle and probably heart tissues, which are major sources of AST rather than the liver. Also, due to this multiple organ distribution of ALP, increased serum activity may be caused by a wide variety of disorders involving several organs. Findings from this study showed that there was no significant change $(p>0.05)$ in the pre- and post-exercise values of both male and female participants. This result, in conjunction with those of ALT and AST indicates a healthy liver function post-exercise.
The LDH test is used to detect tissue damage and as an aid in the diagnosis of heart attack and liver disease [18]. Although, the post-exercise results for male participants showed a significant increase over the pre-exercise results, there was a decrease in the post exercise result when compared with the pre-exercise results for female participants, however, this decrease was not statistically significant. The increase in LDH levels for the male participants may be due to increased damage to muscle cells following the exercise regimen, which may not have occurred in the women.

The post-exercise GGT (gamma-glutamyl transferase) results obtained for male participants showed a non-significant $(p>0.05)$ decrease. Meanwhile, there was an insignificant increase in the post-exercise value for female participations. These results are in agreement with the GGT values reported by Statland et al [19] and Ayca et al [20]. Thus, agreeing with earlier results obtained for ALT, AST, ALP and $\mathrm{LDH}$, indicating a healthy liver post-exercise.

The result for total protein concentration (Tables 3 and 4) showed that there was a decrease in the post-exercise value of both male and female participants when compared with their preexercise values. However, while the decrease in the male participants was statistically significant, the decrease in the female participants was not statistically significant. A plausible reason for this decrease in total protein value has been suggested [21-23], to be due to increased demand of glucogenic amino acids for the generation of energy leading to increased degradation of proteins. A similar result was obtained for plasma albumin. Bilirubin, which is a yellowish coloured product of hemoglobin degradation is a marker for hepatobiliary injury, especially cholestasis [24]. Also, in acute human hepatic injury, total bilirubin can be a better indicator of disease severity compared to ALT [25]. Bilirubin may also be increased due to nonhepatic causes such as hemolysis. Therefore, the non-significant changes observed in all bilirubin values (i.e., total, direct and indirect bilirubin), which were within normal physiological range, could be due to the fact that the exercise regimen did not cause liver injury.

\section{CONCLUSION}

The results obtained indicates that a 6-week physical and flexibility exercise programme did not cause any adverse change in liver and serum parameters as they remained within reference range. 


\section{ACKNOWLEDGEMENT}

The authors thank all the subjects for their voluntary participation in this study.

\section{REFERENCES}

1. Sheri R, Colberg RJ, Sigal B, Fernhall JG, Bryan JB, Rubin RR, Chasan-taber L, Albright A, Barry B. Exercise and Type 2 Diabetes. Diabetes Care 2010; 33: 147167.

2. Thompson PD, Buchner D, Pina IL, Balady GJ, Williams $M A$, Marcus BH. Exercise and physical activity in the prevention and treatment of atherosclerotic cardiovascular disease: A statement from the council on clinical cardiology (subcommittee on exercise, rehabilitation, and prevention) and the council on nutrition, physical activity, and metabolism (subcommittee on physical activity). Circulation 2003; 107: 3109-3116.

3. Wilmore J, Knuttgen H. Aerobic Exercise and Endurance Improving Fitness for Health Benefits. The Physician and Sports Med 2003; 31: 45.

4. O'Connor $D$, Crowe $M$, Spinks W. Effects of static stretching on leg capacity during cycling. Turin 2005; 46 : 52-56.

5. De Vos N, Singh N, Ross D, Stavrinos T. Optimal Load for Increasing Muscle Power During Explosive Resistance Training in Older Adults. J Gerontol 2005; 60(a): 638-647.

6. Janssen, I. and LeBlanc, A. Systematic review of the health benefits of physical activity and fitness in schoolaged children and youth. Int $J$ Behavioural Nutr and Physical Activity 2010, 7 (40): 1-16.

7. American College of Sports Medicine position stand. The recommended quantity and quality of exercise for developing and maintaining cardiorespiratory and muscular fitness, and flexibility in healthy adults. Med Sci Sports Exerc 1998; 30: 975 - 991.

8. Coyle EF, Martin WD, Sincore DR, Joyner MJ, Hagberg JM, Holloszy JO. Time course of loss of adaptations after stopping prolonged intense endurance training. $J$ Applied Physiol 1984; 57: 1857- 1864.

9. World Health Organisation. The use and interpretation of anthropometry. Geneva: WHO; World Health Org 1995; 64: 929-941.

10. Kraemer WJ, Ratamess NA, French DN. Resistance training for health and performance. Current Sports Med Reports 2002; 1: 165-171.

11. Brancaccio P, Giuseppe L, Nicola M. Biochemical markers of muscular damage. Clin Chem Lab Med 2010; 48:757-767.

12. Hammouda O, Chtourou H, Chahed H. Diurnal Variations of Plasma Homocysteine, Total Antioxidant Status, and Biological Markers of Muscle Injury During Repeated Sprint: Effect on Performance and Muscle
Fatigue-A Pilot Study. Chronobiol Int 2011; 28(10): 958-967.

13. Bray GA. Don't throw the baby out with the bathwater. Am J Clin Nutr 2004; 70: 347-349.

14. Devries MC, Imtiaz A, Samjoo IA, Hamadeh JM, Tarnopolsky AM. Effect of Endurance Exercise on Hepatic Lipid Content, Enzymes, and Adiposity in Men and Women. Obesity 2008; 16: 2281-2288.

15. Sreenivasa $B C$, Alexander $G$, Kalyani $B$, Pandey $R$, Rastogi S, Pandey A, Choudhuri G. Effect of exercise and dietary modification on serum aminotransferase levels in patients with nonalcoholic steatohepatitis. J Gastroenterol and Hepatol 2006; 21:191-198.

16. Nuri R, Mahmudieh B, Akochakian M, Moghaddasi M. Effect of 15 weeks combination exercise training on lipid profile and fatty liver indices in postmenopausal women with breast cancer. Braz J Biomotricity 2012; 6:297-303.

17. Leibowitz A, Klin $Y$, Gruenbaum BF, Gruenbaum SE, Kuts R, Dubilet M, Ohayon S, Boyko M, Sheiner E, Shapira $Y$, et al. Effects of strong physical exercise on blood glutamate and its metabolite 2-ketoglutarate levels in healthy volunteers. Acta Neurobiol Exp 2012; 72: 385-396.

18. David JS. Laboratory Test Handbook, ed 4. New York, Lexi Comp Inc, 1996.

19. Statland BE, Winkel $P$, Bokelund $H$. Factors contributing to intra-individual variation of serum constituents: 2 . Effects of exercise and diet on variation of serum constituents in healthy subjects. Clin Chem 1973; 19: 1380-1383.

20. Ayca B, Sener A, Ramazanoglu N, Oba R. The effect of competition on gamma-glutamyl transferase, creatinine and protein levels of taekwondo players. Afri J Pharm Pharmacol 2012; 6: 1462-1468.

21. Dreyer HC, Fujita S, Cadenas JG, Chinkes DL, Volpi E, Rasmussen BB. Resistance exercise increases AMPK activity and reduces $4 E-B P 1$ phosphorylation and protein synthesis in human skeletal muscle. J Physiol 2006; 576: 613-624.

22. Rose AJ, Alsted TJ, Jensen TE, Kobberø JB, Maarbjerg SJ, Jensen J, Richter EA. A Ca (2+)-calmodulin-eEF2KeEF2 signalling cascade, but not AMPK, contributes to the suppression of skeletal muscle protein synthesis during contractions. J Physiol 2009; 587: 1547-1563.

23. Dideriksen K, Reitelseder S, Holm L. Influence of Amino Acids, Dietary Protein, and Physical Activity on Muscle Mass Development in Humans. Nutrients 2013; 5: 852876.

24. Ozer J, Ratner M, Shaw M, Bailey W, Schomaker S. The current state of serum biomarkers of hepatotoxicity. Toxicol 2008; 245: 194-205.

25. Dufour DR, Lott JA, Nolte FS, Gretch DR, Koff RS, Seeff LB. Diagnosis and monitoring of hepatic injury. II. Recommendations for use of laboratory tests in screening, diagnosis, and monitoring. Clin Chem 2001; 47: 1133-1135. 